\section{S. Kaihara}

Director, National Okura Hospital, Tokyo, Japan

\section{Early Expectations}

\section{Japan}

In the late 1960 s, when mainframe computers were introduced in universities, there were only few physicians who foresaw the future possibilities of computers for medical research and patient care. When we try to categorize these "visionary" physicians, we could discern three groups of them, namely, (1) those who believed in the contributions of computer processing of biosignals, (2) those who thought that in the future computers could replace the physician's decision making, and (3) those who wanted to use computers for daily hospital operations. Most of the early developments were experimental, since it was not possible to install mainframes in the real clinical setting. However, a scientist who successfully applied discriminant analysis to the differential diagnosis of liver diseases implied that in the future a computer could become superior to physicians.

By the year 1980, when MEDINFO 80 was held in Tokyo, the three groups

\footnotetext{
This essay does not describe the full history of medical informatics in Asia, nor does it review the current Asian medical informatics projects; reviewing is beyond my ability and the contents would then certainly be beyond the available space. This contribution represents my personal view, of primarily, the Japanese medical informatics situation with, in addition, some comments on developments in other Asian countries.
}

\title{
The Promise
}

\section{The Promise of Medical Informatics in Asia}

had grown in size as well as in number. At the conference they were exposed to the results of similar developments elsewhere in the world and were encouraged to apply the results of their experimental studies to real clinical settings. A scientific group was formed in 1980 , which in 1984 became the Japan Association of Medical Informatics.

In the area of hospital information systems (HISs), coinciding with the same period, hospitals' financial investments were made to improve the time-consuming insurance claims in Japan, using information technology. According to the Japanese insurance scheme, patients must pay the fee of their visit to the hospital on-site. This rulecontributed much tothe introduction of information technology in hospitals and the development of sophisticated HISs. By the year 1990, almost $100 \%$ of the hospitals in Japan had installed some kind of information system for the processing of insurance claims. But at this stage, medical personnel had not yet participated in the use of a HIS and were still writing their laboratory orders or drug prescriptions on a slip of paper.

A successful order-entry type of HIS was installed in the early 1990 s. At first, physicians were reluctant to use computer terminals but currently almost all university hospitals have a HIS. Approximately $10 \%$ of the hospitals in Japan now have an order-entry systeminstalled.
In the area of biosignal and image analysis, the processing of ECGs,EEGs, and radiological images were the subject of interest. The results of developments in these areas were integrated in automated health check-up systems. Such health check-up systems were well appreciated in the early days but are now gradually being absorbed by hospitals.

In the field of medical decision analysis, patient data were collected from different specialties such as neurology, cardiology, gastroenterology, etc. and were analyzed using various methods, such as statistical methods, artificial intelligence-based techniques, neural networks, etc. The results were well accepted if applied to the original patient data on the basis of which the decision-support systems were developed, but applications in the real clinical setting were almost absent. This is mainly due to the fact that the clinical situation deviated from an experimental setting.

In the beginning of the 1990s, a fourth group of potential users emerged, interested in the application of electronic image transmission, to support medical care in remote areas. By that time, the progress of electronic data transmission technology had enabled such endeavors. This was the beginning of telemedicine in Japan. In the early days, still images were mainly transmitted fromremote islands to main hospitals.

Yearbook of Medical Informatics 1999 


\section{Asian Countries}

Other Asian countries, Australia, New Zealand and Singapore seemed to have been confronted with almost the same situation as that of Japan. In the People's Republic of China, the Chinese Medical Informatics Association (CMIA) was founded in 1983 by Dr. Z. Ouyang. CMIA became the focal point for the medical informatics development in China. In Korea, the Korean Medical Informatics Society was formed by Prof. C.S. Koh in 1989, which also much stimulated the activities of medical informatics. The Hong Kong Society of Medical Informatics, founded by Dr. Tan was another center of medical informaticsactivities in Asia.

In 1989, MEDINFO 89 was held in both Beijing and Singapore. The interest in informationtechnologies in the Asian countries were further stimulated by this world conference. MEDINFO 89 showed that information technology is applicable in health care, not only in developed countries but also in developing countries. Many results of ongoing developments were presented at this conference.

\section{Current Situation or Outcome}

\section{Japan}

Another 10 years have passed since MEDINFO 89 and we are now standing at the threshold of the new century. Have the expectations that we had in mind in the early days been realized? Generally speaking, the computerenvironment surrounding us has changed dramatically, but the essence of health care may not have been influenced by information technology to the degree that was expected.

In hospitals, physicians and nurses are using computers for their daily hospital operations. The computer processes the data and the results are shown on screens in a tabulated or graphical format. But the care given to the patients may be identical to the care given in the 1970s. Physicians do not routinely use computers when making critical decisions in patient care, perhaps because the terminal located in the ward is not connected to any knowledgebase, for instance available on the Internet. Physicians may still have to walk to a terminal in the library, which is connected to MEDLINE, but they are usually too busy to do so.

Sophisticated decision-support systems were developed, but most of them could not be applied to practical problems. Standards for medical knowledge representation are still under development and a knowledge base developed in one institute cannoteasily be used in other institutes. For instance, the expert group ESTRI (Electronic Standard for Transfer of Regulatory Information) operates in the framework of the International Conference on Harmonization of Technical Requirements for Registration of Pharmaceuticals for Human Use, usually abbreviated as ICH. ESTRI has attempted for the past 5 years to set up a standard to electronically transfer the information on adverse drug events on an international level. However, because of the lack of a generally accepted standard method, ESTRI encountered many difficulties to reach consensus among international communities. This attempt is an example of the difficulty to solve a real world problem, even if sophisticated information technology is available.

General practitioners (GPs) who refer patients to a hospital still write a referral letter to a specialist in a hospital and receive in return a hand-written report from the GP, although both physicians use a computer for their daily operations, and the hospital database contains all patient data in its HIS. Again, this is because patient record data are not yet standardized and are, hence, not compatible with each other.
In contrast to information contained in knowledge bases, for patient records there are now methods to standardize the patient record data format. However, the clinical specialties are so diversified that it is thought to be too time-consuming to develop a consensus among the different medical communities.

The lack of common standards to describe clinical data also presents a problem to collect clinical research data from multiple medical institutions. Evidence-based medicine is only possible when reliable clinical databases are available. Medical informatics, at least in Japan, is not yet sufficiently contributing to the development of such databases.

Supporting patient care by image transmission is now attracting the interest of the medical community as well as the public. It was expected that by electronic transmission the medical care in remote areas would be dramatically improved. Many project confirmed this expectation. However, the survey I made on this subject in 1996 showed that two third of the projects had been terminated or were being terminated at the time of the survey. The main reasons for termination were the lack of funding and of personnel. Because the projects were usually funded by research grants or time-limited budgets, discontinuation of the funding implied the end of the project. Some projects used very sophisticated technologies such as highresolution transmission and display of images, which was of interest during the experiment, but too costly to be implemented in real clinical settings. The projects that survived used rather simple and cheap technologies, but even in these projects, the personnet engaged were more or less participating as volunteers. Institutionalized telemedicine projects were very rare.

Image transmission technologies were also of interest at universities to offer students outside the campus 
distance learning. In 1996, the Ministry of Education started a project for University Hospitals, called Medical Information Systems via Communication Satellite, abbreviated as MINCS. The resulting system was the first digital high-definition television communication system with data encryption. Eight national university hospitals participated in this project and are periodically exchanging lectures. A similar system, using an ordinary NTSC television system and ground communication lines, is now in operation at the National Cancer Center in Tokyo and other provincial cancer centers.

In 1997, the IMIA Working Group4 conference on security was held in Osaka, hosted by the Japanese Association for Medical Informatics. This stimulated the interest in security in medical data transmission in Japan since, by the increase of data transmission on the Internet, data protection issues had become a common concern of the people engaged in the development and use of medical information systems. The recommendations were well appreciated in the Japanese medical community.

In spite of the interest in data security, we still do not have any standard solution in Japan. Encryption, electronic signatures, private and public keys, firewalls, etc. are discussed daily. It may be possible to implement as many security protocols as possible, but no one knows whether the operation of the systems can be maintained after the implementation, since we have simply not accumulated sufficient experience in using such technologies in a real setting. I will give just one example. A general practitioner who referred a patient to a hospital may want to access the actual patient data in the HIS database, using a computer terminal located in his office, connected to the HIS by telephone. What, then, is the security method to be used, which is acceptable in terms of cost and operability? Do we have an answer for this question?

\section{Asian Countries}

We have said that information technology contributes not only to developed countries but also to developing countries. Nowadays, wherever one goes in Asia, very sophisticated information systems can be found in several major medical institutions. In this sense, information technology is now available all over the world and in this respect Asian countries do not differ. However, when visiting a remote clinic, one may be confronted with a situation that information, indispensable for daily care, is lacking. In some countries in Asia, the difference in available information between cities and remote areas is considerable. The contribution of information technology todeveloping countries should not increase the above disparity but decrease it. I hope that in the future there will be suitable information technology for all areas, remote or not. Unfortunately, at present it is not yet fully developed. Satellite communication may be a good candidate for making information available, since it may be far cheaper than ground transmission cables to remote areas. Also, very simple pocket electronic devices may be developed for the substitution of paper books. I hope that our interest in this direction will increase.

\section{Promises for the Future}

\section{Medical Informatics as a Basis for Medical Science}

Medical informatics is a science that is able to provide a basis for the science of medicine. "Basis for the science of medicine" may include several components, for instance, to offer physicians a standard method to describe medical concepts, knowledge and clinical data, a standard method to process medical data so that clinicians can make rational decisions at the proper time, or a standard method for transferring data to the required location at the proper time. The results of medical informatics, in this respect, must be applied ultimately to the real clinical setting. The users of the results of R\&D in medical informatics, are physicians, nurses, technicians and many others. The wording "standard method" does not necessarily mean the most advanced and sophisticated methodology. It should be a method that is applicable in real settings in terms of availability, cost and easy operability. In some fields, we must develop such a methodology ourselves. But in other fields, a standard method may often already exist but, because of lack of consensus to adopt it or because of ignorance of its value, the method is not used to its full extent. In such situations, the role of medical informaticians may be to lead the users in the field to a consensus to adopt the method.

If we consider the role of medical informatics in the way mentioned above, there is a huge open field where medical informatics can contribute in the future. To play such a role, however, medical informaticians must be able to communicate with people in other medical specialties through the methodology we already have, and if we do not yet have an appropriate method we should work hard developing it, even if such a method is not so interesting from the viewpoint of advanced information science. The value of medical informatics is not necessarily in the advancement but also in the applicability to the real world. For instance, developing consensus for the structure and the terms of patient records may be tremendously cumbersome, but only the people in medical informatics can give directions in this field. Development of a large-scale multi-institutional database of clinical data to find "evidence" for a 
certain clinical problem is another example of time consuming but important work. More examples can be given.

I am pleased to say that the contribution of medical informatics to health care is gradually increasing. Consensus is being developed in the field of electronic patient records, telemedicine, knowledge representation, and data protection. At present, the consensus is still piecemeal, but will be integrated after repeated attempts. A promising project is an image reference center development project at the National Cancer Center in Tokyo and the National Cardiovascular Disease Center in Osaka, which is a part of a G7 health care application project. The purpose of this project is to accumulate valuable medical images such as $\mathrm{X}$-ray pictures or histological images from all over the world and provide them to appropriate institutions in the world, using Internet. The consensus about the format of the images etc. is being developed and, hopefully, it will contribute to global health care.

\section{Patient Participation}

Another important aspect of medical informatics in the future is the contribution of patients themselves. Although apatientisa partner in medical care, the information systems developed in the past are mainly intended to be used by care providers. In Japan, some hospitals are now planning to install a HIS that has terminals at the bedside for the patient's own use. Patients have two types of needs with respect to required information. First, they need information, for instance, on the care they receive, such as the scheduling of clinical tests, information on their stay in the hospital, or on entertainment, such as TV programs. Second, they want to communicate with various groups of people such as nurses, their family at home, or colleagues at the office. The terminal at the bedside will meet all the above informationneeds. Telemedicine for home care is another example of patient participation. Patients communicate with their physician from their home to receive instructions. Alsogeneral practitioners and community nurse stations plan to install such a system for daily use. During the time in between their visits to the patient at home, physicians and nurses can still communicate with the patients when necessary.

Regarding continuing education, satellite communication will be merged with ground communication lines, to offer medical practitioners lectures given by universities or during conferences. MINCS, mentioned above, is also being expanded to nonuniversity clinics.

\section{Asian Countries}

As stated in the beginning, it is beyond my ability to review all the medical informatics projects in Asian countries, but a very good view of Asian projects was given during MEDINFO 98 in Seoul, which was well attended by Asian researchers in medical informatics. In reviewing the Proceedings, I was pleased to see many interesting as well as practical projects being conducted in many Asian countries.

In Asia, there are currently seven medical informatics societies that together form APAMI (Asian-Pacific Medical Informatics) under the leadership of Dr.K.C.Lun, organizing conferences in the same pace as MEDINFO. The number of members in each society has also grown to hundreds or even thousands of members in some countries. This growing number seems to go in parallel to the growing activities in all countries.

Even in non-IMIA member countries, medical informatics is getting its essential position in health care. I am personally involved in the project of Cho-Ray Hospital at Ho Chi Ming City in Vietnam, in which a small-scale system, but containing a local area network, is being implemented in the hospital. Local people developed the system with local equipment and this system will certainly prove to be valuable and will contribute to the hospital as well as the surrounding community.

In summary, reviewing the future of medical informatics in Asia, there i still a long way to go, but the future is certainly very promising.

\author{
Address of the author: \\ Shigekoto Kaihara, M.D., \\ Director, National Okura Hospital, \\ 2-10-1 Okura, \\ Setagaya-ku, \\ Tokyo 157-0074, \\ Japan \\ E-mail: kaihara-tky@umin.ac.jp
}

